\title{
Calculation of electric field and optical transitions in InGaN/GaN quantum wells
}

\author{
Ursula M. E. Christmas, ${ }^{\text {a) }}$ A. D. Andreev, and D. A. Faux \\ Advanced Technology Institute, University of Surrey, Guildford, GU2 7XH, United Kingdom
}

(Received 26 May 2005; accepted 30 August 2005; published online 12 October 2005)

\begin{abstract}
We present analytical expressions for internal electric field and strain in single and multiple quantum wells, incorporating electromechanical coupling, spontaneous polarization, and periodic boundary conditions. Internal fields are typically $2 \%$ lower than the fields calculated using an uncoupled model. We point out two possible interpolation routes to calculate the piezoelectric (PZ) constants $e_{i j}$ of an alloy from the PZ constants of the constituent materials and show that, for an $\mathrm{In}_{0.2} \mathrm{Ga}_{0.8} \mathrm{~N} / \mathrm{GaN}$ quantum well system, the respective internal electric fields differ by $10 \%$. Using an effective-mass model, we explore the effect of the uncertainty in the elastic and PZ constants of $\mathrm{GaN}$ on the internal field and optical transitions of InGaN/GaN quantum wells, and find that the range of published values of $e_{i j}$ produces an uncertainty of more than $\pm 20 \%$ in the internal field and of more than $\pm 30 \%$ in the blueshift in optical transition energy between zero bias and flatband conditions (when the applied field is equal and opposite to the internal field). Using the PZ constants of Shimada et al. [J. Appl. Phys. 84, 4951 (1998)] in our model gives the best fit to results in the literature for internal field and optical transition energy in InGaN/GaN quantum wells. We find that a well with a smooth In gradient along the growth direction has similar optical properties to a well with constant composition, if the average In content of the two wells is the same. (C) 2005 American Institute of Physics. [DOI: 10.1063/1.2077843]
\end{abstract}

\section{INTRODUCTION}

There has been much research recently in the field of III-nitride semiconductors, and InGaN quantum wells (QWs) in particular, because of their wide band gap, which means they can emit blue or violet light. Short-wavelength lasers allow a high density of information to be stored on a digital versatile disk (DVD) and InGaN QW light-emitting diodes (LEDs) can be used to excite a mixture of phosphors, producing white light. ${ }^{1}$

The wavelength of the light emitted from a III-nitride QW depends not only on the band gap but also on the large internal electric field due to piezoelectric (PZ) and spontaneous polarization. ${ }^{2}$ The $\mathrm{PZ}$ field arises from strain due to the lattice mismatch between the $\operatorname{In}_{x} \mathrm{Ga}_{1-x} \mathrm{~N}$ well and the GaN barriers (about $2 \%$ for $x=0.2$ ) and causes redshift in the optical transition energy. ${ }^{3}$ Spontaneous polarization (SP) is a feature of the III-nitride wurtzite crystal even when there is no strain present. In a typical $\mathrm{InGaN} / \mathrm{GaN}$ device, $\mathrm{SP}$ is an order of magnitude smaller than PZ polarization. When a reverse bias is applied, a blueshift is seen due to the partial cancellation of the internal field. ${ }^{4-8}$ By measuring the blueshift as a function of applied field it is possible to infer the size of the internal field.

The existence of the converse PZ effect (whereby an electric field gives rise to stress) means that we should use coupled equations to calculate the strain and internal field, or at least explore whether electromechanical coupling makes a significant difference to the result. Pan and Tonon ${ }^{9}$ and $\operatorname{Pan}^{10}$ have developed a coupled model for quantum dots but, un-

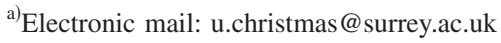

like the present work, it does not incorporate SP. Bernardini and Fiorentini ${ }^{2}$ explored the application of periodic boundary conditions to multiple quantum wells; in this paper we go further and apply periodic boundary conditions to the coupled model.

There is much uncertainty ${ }^{11}$ in the values of the material parameters used to describe the internal field of $\mathrm{GaN}$ and InN, namely, the PZ tensor, the elastic stiffness tensor $C_{i j k l}$, and SP. This is partly because the study of III-nitrides is in its infancy, but also because every sample has different defects and because it is difficult to grow large enough crystals. Then there is the separate problem of estimating the corresponding properties of the alloy.

The uncertainty in the material parameters gives rise to uncertainty in the optical transition energy. Experiments on the optical properties of $\mathrm{InGaN} / \mathrm{GaN}$ QWs have been carried out by Takeuchi et al., ${ }^{4}$ Lai et al., ${ }^{6}$ Jho et al., ${ }^{7}$ Waltereit et al. ${ }^{12}$ Feng et al.,${ }^{13}$ Dhar et al.,${ }^{14}$ Wang et al. ${ }^{15}$ and others; theoretical studies have been done by Oriato and Walker, ${ }^{16}$ and Xiao and Kim. ${ }^{17}$ The results vary widely, even for similar well widths and In fractions. The motivation for the present work is to explore the reasons for these variations.

Another problem is to ascertain the properties of a particular device. For example, the In fraction $x$, which is usually estimated indirectly, is a strong function of the growth temperature. At the end of the growth of a well, the temperature is often increased in order to grow the GaN barrier and this may cause loss or redistribution of In. In this paper we investigate the effect on the optical transition energy of an In gradient along the growth axis.

In short, this paper explores some of the uncertainties contributing to the calculation of the internal field (and con- 
sequently the optical transition energy) in InGaN/GaN QWs. This article is organized as follows. Section II contains the theory, with Sec. II A presenting our analytic solution to the electromechanically coupled equations for strain and internal field in a QW, and Sec. II B the application of this coupling to the periodic boundary conditions appropriate for multiple quantum wells (MQWs). Section II C outlines our model for calculating electronic structure and, in Sec. II D, we point out two different routes for calculating the PZ tensor elements $e_{i j k}$ of an alloy by interpolation. Section III contains results. In Sec. III A we show that electromechanical coupling produces an internal field $2 \%$ lower than that predicted by uncoupled equations. Section III B shows that the two alternative routes for calculating the PZ constants of InGaN produce internal fields that differ by around $10 \%$ and investigates which route agrees better with experimental results. In Sec. III D we explore the effect of the uncertainties in the values of $C_{i j k l}$ and of the PZ tensor on the calculation of internal field and optical properties; we find that uncertainties in the PZ constants $e_{i j}$ give rise to an uncertainty of the order of $\pm 20 \%$ in internal field, $\pm 5 \%$ in the ground-state electronic transition energy $E_{\mathrm{tr}, 0}$ at zero bias and $\pm 30 \%$ in blueshift (which is the difference between the ground-state transition energy at zero bias and the maximum ground-state transition energy, when the applied field is equal and opposite to the internal field). In Sec. III E we compare our calculations of internal field, $E_{\mathrm{tr}}$ and blueshift with the published results; we show that the PZ constants calculated by Shimada et al. ${ }^{18}$ give the best correspondence with experimental results, and we discuss some of the reasons for the wide variation in experimental and theoretical results. In Sec. III F we investigate the effect of a smooth In gradient in the growth direction and show that it does not have a significant effect on the optical transition energy. In Sec. IV we draw our conclusions.

\section{THEORY}

\section{A. Quantum wells}

We now present our fully coupled equations for internal electric field and strain in the growth direction in a wurtzite QW or MQW system in the (0001) orientation. "Coupled" means that the two-way piezoelectric interaction between strain and electric field is taken into account. Spontaneous polarization is also incorporated (Pan and Tonon ${ }^{9}$ and $\operatorname{Pan}^{10}$ models incorporate electromechanical coupling but do not incorporate $\mathrm{SP}$ ).

Stress is given by

$$
\sigma_{i}=C_{i j} \epsilon_{j}-F_{k} e_{k i}
$$

where we use the Voigt notation $(i=1 \ldots 6$ and $j=1 \ldots 6)$, in which the first three elements of $\sigma$ (stress) and $\epsilon$ (strain) denote the principal stresses (strains) and the last three denote the shear elements; $C_{i j}$ are the elastic stiffnesses and the $e_{k i}$ are the PZ constants. $F_{k}$ is the $k$ th component of the internal electric field $(k=1 \ldots 3)$ and the second term represents the converse PZ effect, whereby an electric field modifies the stress field. The Einstein convention is used, in which repeated indices imply summation.
In a wurtzite crystal such as GaN the $C_{i j}$ and $e_{k i}$ take the following form:

$$
\begin{aligned}
C_{i j} & =\left(\begin{array}{cccccc}
C_{11} & C_{12} & C_{13} & 0 & 0 & 0 \\
C_{12} & C_{11} & C_{13} & 0 & 0 & 0 \\
C_{13} & C_{13} & C_{33} & 0 & 0 & 0 \\
0 & 0 & 0 & C_{44} & 0 & 0 \\
0 & 0 & 0 & 0 & C_{44} & 0 \\
0 & 0 & 0 & 0 & 0 & \frac{C_{11}-C_{12}}{2}
\end{array}\right) \\
e_{k i} & =\left(\begin{array}{cccccc}
0 & 0 & 0 & 0 & e_{15} & 0 \\
0 & 0 & 0 & e_{15} & 0 & 0 \\
e_{31} & e_{31} & e_{33} & 0 & 0 & 0
\end{array}\right) .
\end{aligned}
$$

In a quantum well with the $z$ axis in the growth direction, the boundary conditions are of zero stress in the $z$ direction, zero shear stresses and strains, and symmetry of $x$ and $y$ directions in the plane of the well. Thus

$$
\begin{aligned}
& \sigma_{1}=\sigma_{2}, \\
& \sigma_{3}=0, \\
& \sigma_{4}=\sigma_{5}=\sigma_{6}=0, \\
& \epsilon_{1}=\epsilon_{2}=\epsilon_{0}, \\
& \epsilon_{4}=\epsilon_{5}=\epsilon_{6}=0 .
\end{aligned}
$$

$\epsilon_{0}$ is the misfit strain between the in-plane lattice parameters $a$ of the well material and of the barrier,

$$
\epsilon_{0}=\frac{a_{\mathrm{GaN}}-a_{\mathrm{InGaN}}}{a_{\mathrm{InGaN}}},
$$

which is about $2.2 \%$ for $\operatorname{In}_{0.2} \mathrm{Ga}_{0.8} \mathrm{~N} / \mathrm{GaN}$. It is assumed that the barrier is fully relaxed (there is normally a lattice mismatch between the barrier and the sapphire substrate on which it is grown, but the strain is relaxed by means of dislocations).

Substituting Eqs. (2)-(4) into Eq. (1), we have

$$
\sigma_{3}=0=2 C_{13} \epsilon_{0}+C_{33} \epsilon_{3}-e_{33} F_{3} .
$$

We then take the well-known electrostatic equation,

$$
D_{m}=\varepsilon_{m k} F_{k}+P_{m},
$$

where $\mathbf{D}$ is the electrostatic displacement, $\varepsilon_{m k}$ is the dielectric tensor, which is diagonal, and $\mathbf{P}$ is the polarization. In the well, the polarization has both piezoelectric and spontaneous components,

$$
\mathbf{P}=\mathbf{P}^{\mathrm{PZ}}+\mathbf{P}^{\mathrm{SP}}
$$

and the PZ polarization is given by

$$
P_{m}^{\mathrm{PZ}}=e_{m k} \epsilon_{k} .
$$

Substituting into Eq. (7) we have, for the $z$ component, 


$$
D_{3}=\varepsilon_{33} F_{3}+2 e_{31} \epsilon_{0}+e_{33} \epsilon_{3}+P_{3}^{\mathrm{SP}} .
$$

$\mathbf{D}, \mathbf{F}$, and $\mathbf{P}^{\mathrm{SP}}$ are entirely in the $z$ direction; other components vanish, so the subscripts are dropped from now on. In the absence of free charges,

$$
\boldsymbol{\nabla} \cdot \mathbf{D}=0
$$

so $D$ must be constant throughout all layers.

Solving Eqs. (6) and (10) for the internal field $F$ and the strain $\epsilon_{3}$, we have

$$
\begin{aligned}
& F_{\text {coup }}=\frac{2 \epsilon_{0}\left(C_{13} e_{33}-C_{33} e_{31}\right)+C_{33}\left(D-P^{\mathrm{SP}}\right)}{C_{33} \varepsilon_{33}+e_{33}^{2}}, \\
& \epsilon_{3, \text { coup }}=\frac{-2 \epsilon_{0}\left(C_{13} \varepsilon_{33}+e_{31} e_{33}\right)+e_{33}\left(D-P^{\mathrm{SP}}\right)}{C_{33} \varepsilon_{33}+e_{33}^{2}},
\end{aligned}
$$

which are the coupled equations for internal field and [0001] strain in wurtzite QWs grown on a (0001) substrate.

In analyzing QWs, it is common to use an uncoupled model, ignoring the converse PZ effect. In this case, the last term is omitted from Eq. (1) but Eq. (10) is unchanged. The solutions for the uncoupled model are therefore

$$
\begin{aligned}
& F_{\text {uncoup }}=\frac{2 \epsilon_{0}\left(C_{13} e_{33}-C_{33} e_{31}\right)+C_{33}\left(D-P^{\mathrm{SP}}\right)}{C_{33} \varepsilon_{33}}, \\
& \epsilon_{3, \text { uncoup }}=-2 \epsilon_{0} \frac{C_{13}}{C_{33}} .
\end{aligned}
$$

As $e_{i j} \rightarrow 0$, Eqs. (12) and (13) simplify to Eqs. (14) and (15).

In the case of a single $\mathrm{QW}$, the electrostatic displacement throughout the system (except near the surface) is equal to the SP in the barrier, ${ }^{2}$

$$
D=P^{\mathrm{SP}, B} \text {, }
$$

and $P^{\mathrm{SP}, B}$ can simply be substituted for $D$ in Eqs. (12)-(15). The electric field in the barriers vanishes.

\section{B. Multiple quantum wells}

In modeling MQWs, we assume that the total potential drop must be less than the band gap. ${ }^{2}$ Bernardini and Fiorentini $^{2}$ approximate this requirement by using the periodic boundary condition,

$$
\sum_{q} l^{(q)} F^{(q)}=0
$$

where the sum runs over all the layers, including barrier layers, and $l$ denotes the thickness of a layer. When Eq. (17) is used with Eq. (7), the $D$ of the whole stack, and hence the $F$ of the $n$th layer, can be determined ${ }^{2}$ as

$$
F_{\text {uncoup }}^{(n)}=\frac{\sum_{q} l^{(q)} P^{(q)} / \varepsilon^{(q)}-P^{(n)} \sum_{q} l^{(q)} / \varepsilon^{(q)}}{\varepsilon^{(n)} \sum_{q} l^{(q)} / \varepsilon^{(q)}},
$$

where $\varepsilon$ is short for $\varepsilon_{33}$ and the subscript indicates that this is based on an uncoupled model. A similar approach can be used with the coupled Eq. (12). We use the shorthand,

$$
\begin{aligned}
& A=2 \epsilon_{0}\left(C_{13} e_{33}-C_{33} e_{31}\right)-C_{33} P^{\mathrm{SP}}, \\
& B=C_{33} \varepsilon_{33}+e_{33}^{2}, \\
& C=C_{33},
\end{aligned}
$$

so Eq. (12) becomes

$$
F_{\text {coup }}=\frac{A+C D}{B} \text {. }
$$

We substitute Eq. (22) into Eq. (17) to find the electrostatic displacement throughout the system,

$$
D=\frac{-\sum_{q} l^{(q)} A^{(q)} / B^{(q)}}{\sum_{q} l^{(q)} C^{(q)} / B^{(q)}} .
$$

Substituting Eq. (23) back into Eq. (22) we have the internal field in the $n$th layer,

$$
F_{\text {coup }}^{(n)}=\frac{A^{(n)} \sum_{q} l^{(q)} C^{(q)} / B^{(q)}-C^{(n)} \sum_{q} l^{(q)} A^{(q)} / B^{(q)}}{B^{(n)} \sum_{q} l^{(q)} C^{(q)} / B^{(q)}} .
$$

We use Eq. (24) from now on in MQW cases.

The strain in the growth direction in the $n$th layer follows from Eqs. (13) and (23),

$$
\epsilon_{3, \text { coup }}^{(n)}=\frac{G^{(n)} \sum_{q} l^{(q)} C^{(q)} / B^{(q)}-e^{(n)} \sum_{q} l^{(q)} A^{(q)} / B^{(q)}}{B^{(n)} \sum_{q} l^{(q)} C^{(q)} / B^{(q)}},
$$

where

$$
\begin{aligned}
& G=-2 \epsilon_{0}\left(C_{13} \varepsilon_{33}+e_{31} e_{33}\right)-e_{33} P^{\mathrm{SP}}, \\
& e=e_{33} .
\end{aligned}
$$

\section{Electronic structure}

The electronic transition energy is calculated using an effective-mass approximation. The wells are shallow compared to the size of the band gap and so it is not necessary to use the full $\mathbf{k} \cdot \mathbf{p}$ model. We calculate the single-particle ground-state electron and hole energies numerically by solving Harrison's ${ }^{19}$ finite difference version [Eq. (28) below] of the one-dimensional Schrödinger equation.

$$
\begin{aligned}
\frac{\psi(z+\delta z)}{m^{*}(z+\delta z / 2)} \simeq & \left\{\frac{2(\delta z)^{2}}{\hbar^{2}}[V(z)-E]+\frac{1}{m^{*}(z+\delta z / 2)}\right. \\
& \left.+\frac{1}{m^{*}(z-\delta z / 2)}\right\} \psi(z)-\frac{\psi(z-\delta z)}{m^{*}(z-\delta z / 2)},
\end{aligned}
$$

where $\psi$ is the wave function, $m^{*}$ is the effective mass, $z$ is the distance in the growth direction, $V$ is the potential, and $E$ is the energy. We search for an energy that satisifies Eq. (28) and the boundary conditions $\psi(z) \rightarrow 0$ and $\partial \psi(z) / \partial z \rightarrow 0$ as $z \rightarrow \pm \infty$. 
TABLE I. Table of material parameters used in our model (in-plane lattice constants $a$, spontaneous polarization $P^{\mathrm{SP}}$, elastic constants $C_{i j}$, PZ constants $d_{i j}$, dielectric constants $\varepsilon_{33, r}$ (where $r$ denotes the relative permittivity), deformation potentials $a_{i}$ and $D_{i}$, bandgap $E_{g}$ (before strain), valence-band offset VBO (before strain), and effective mass $m^{*}$ ). $b$ is the bowing parameter. All are taken from the review paper of Vurgaftman and Meyer (Ref. 11) except where indicated.

\begin{tabular}{|c|c|c|}
\hline Parameter & $\mathrm{GaN}$ & InN \\
\hline$a(\mathrm{~nm})$ & 0.3189 & 0.3545 \\
\hline$P^{\mathrm{SP}}\left(\mathrm{C} \mathrm{m}^{-2}\right)$ & -0.034 & -0.042 \\
\hline$b\left(P^{\mathrm{SP}}\right)\left(\mathrm{C} \mathrm{m}^{-2}\right)$ & \multicolumn{2}{|c|}{-0.037} \\
\hline$C_{11}(\mathrm{GPa})$ & 390 & 223 \\
\hline$C_{12}(\mathrm{GPa})$ & 145 & 115 \\
\hline$C_{13}(\mathrm{GPa})$ & 106 & 92 \\
\hline$C_{33}(\mathrm{GPa})$ & 398 & 224 \\
\hline$d_{31}(\mathrm{pm} / \mathrm{V})$ & -1.6 & -3.5 \\
\hline$d_{33}(\mathrm{pm} / \mathrm{V})$ & 3.1 & 7.6 \\
\hline$\varepsilon_{33, r^{\mathrm{a}}}$ & 10.28 & 14.61 \\
\hline$a_{1}(\mathrm{eV})$ & -4.9 & -3.5 \\
\hline$a_{2}(\mathrm{eV})$ & -11.3 & -3.5 \\
\hline$D_{1}(\mathrm{eV})$ & -3.7 & -3.7 \\
\hline$D_{2}(\mathrm{eV})$ & 4.5 & 4.5 \\
\hline$D_{3}(\mathrm{eV})$ & 8.2 & 8.2 \\
\hline$D_{4}(\mathrm{eV})$ & -4.1 & -4.1 \\
\hline$E_{g}(\mathrm{eV})$ & 3.51 & 0.78 \\
\hline$b\left(E_{g}\right)(\mathrm{eV})$ & \multicolumn{2}{|c|}{1.4} \\
\hline VBO (eV) & & 0.5 \\
\hline$m_{e}^{*}\left(m_{0}\right)$ & 0.2 & 0.07 \\
\hline$m_{h h}^{\perp}\left(m_{0}\right)^{\mathrm{b}}$ & 1.02 & 1.25 \\
\hline
\end{tabular}

${ }^{2}$ Reference 41.

${ }^{\mathrm{b}}$ Reference 42 .

We neglect exciton binding energies because they are typically less than $25 \mathrm{meV} .^{20,21} \mathrm{We}$ have assumed that the field due to doped regions is negligible. This is because at room temperature only about $1 \%$ of acceptors are ionized, due to the strong binding energy between the $\mathrm{Mg}$ dopant and GaN. Thus in a typical device the $p$ - $n$ field would be some three orders of magnitude smaller than the PZ field.

An internal field causes electrons and holes to congregate at opposite boundaries of the well, setting up their own opposing field, which becomes significant if the carrier density is high. We have chosen to ignore this effect and concentrate on the pure PZ/SP field, so that in analyzing our results there is no confusion between various competing effects. Thus we operate in a low carrier density regime, less than $10^{11} \mathrm{~cm}^{-2}$, which would produce a field of the order of $10^{-2} \mathrm{MV} \mathrm{cm}^{-1}$, at least 100 times smaller than the PZ field and therefore negligible.

We use the material parameters set out in Table I.

\section{Two different interpolation routes for calculating the PZ tensor of an alloy}

In order to calculate the internal field from the strain in the well, we need to know the PZ constants of the alloy in the well. This is normally achieved by linear interpolation between the properties of $\mathrm{InN}$ and $\mathrm{GaN}$ (although the firstprinciples calculations of Al-Yacoub and Bellaiche ${ }^{22}$ show that $\mathrm{Ga}_{0.5} \operatorname{In}_{0.5} \mathrm{~N}$ consisting of an ordered system of monolayers of alternating $\mathrm{Ga}$ and In has PZ constants a few percent
TABLE II. Comparison of results from electromechanically coupled and uncoupled models, respectively. $F$ is internal electric field, $E_{\mathrm{tr}, 0}$ ground-state electronic transition energy (with no applied bias), and $\Delta E_{\mathrm{tr}}$ blueshift (difference between transition energy with no applied bias and that with an applied field equal and opposite to internal field). Results are presented for (a) for a single $3 \mathrm{~nm} \mathrm{In} 0.2 \mathrm{Ga}_{0.8} \mathrm{~N} / \mathrm{GaN} \mathrm{QW}$ and (b) for the $\mathrm{In}_{0.2} \mathrm{Ga}_{0.8} \mathrm{~N} / \mathrm{GaN}$ MQWs depicted in Fig. 1.

\begin{tabular}{|c|c|c|c|}
\hline \multirow{2}{*}{\multicolumn{2}{|c|}{ (a) }} & Uncoupled & Coupled \\
\hline & & \multicolumn{2}{|c|}{ Single QW } \\
\hline & $F\left(\mathrm{MV} \mathrm{cm}^{-1}\right)$ & -3.90 & -3.81 \\
\hline & $E_{\mathrm{tr}, 0}(\mathrm{eV})$ & 2.27 & 2.29 \\
\hline & $\Delta E_{\mathrm{tr}}(\mathrm{meV})$ & 695 & 670 \\
\hline \multirow[t]{4}{*}{ (b) } & & \multicolumn{2}{|c|}{ MQWs } \\
\hline & $F\left(\mathrm{MV} \mathrm{cm}^{-1}\right)$ & -3.05 & -2.98 \\
\hline & $E_{\mathrm{tr}, 0}(\mathrm{eV})$ & 2.51 & 2.53 \\
\hline & $\Delta E_{\mathrm{tr}}(\mathrm{meV})$ & 450 & 435 \\
\hline
\end{tabular}

smaller than the corresponding interpolated values). We now discuss the possible interpolation routes for finding the PZ constants of an alloy.

Equation (9) uses $e_{i j}$ to relate strain to polarization. There is an alternative PZ tensor, which is defined in terms of stress $\sigma_{j}$,

$$
P_{i}^{\mathrm{PZ}}=d_{i j} \sigma_{j} \text {. }
$$

The $d_{i j}$ are related to the $e_{i j}$ by

$$
e_{i j}=d_{i k} C_{k j},
$$

where $C_{k j}$, as before, represents the elastic stiffness. In our calculations we use $e_{i j}$ because strain is simpler to calculate than stress. Since uncertainty surrounds the values of both $d_{i k}$ and $C_{k j}$, the uncertainty of $e_{i j}$ (unless is it measured directly) is compounded. However, no direct literature values of $e_{i j}$ for InN appear to be available, so we must rely on the $d_{i j}$ calculated by Bernardini and Fiorentini (Ref. 23) and, for GaN, the $d_{i j}$ is more often reported ${ }^{23-26}$ than the $e_{i j} .{ }^{18,27}$

The equation for interpolation between material properties of $\mathrm{GaN}$ and $\mathrm{InN}$ is ${ }^{28}$

$$
Y\left(\operatorname{In}_{x} \mathrm{Ga}_{1-x} \mathrm{~N}\right)=x Y(\operatorname{InN})+(1-x) Y(\mathrm{GaN})-b x(1-x),
$$

where $Y$ is any material property and $b$ is a bowing parameter, which is usually taken to be zero in the case of the PZ and elastic properties, but not in the case of the bandgap ${ }^{28}$ and $\mathrm{SP}^{29}$

To estimate the $e_{i j}$ of InGaN, there are two possible routes. We can calculate $e_{i j}$ of pure $\mathrm{GaN}$ and of InN using Eq. (30), and then use Eq. (31) (the " $e$-first" route). Or we can use Eq. (31) to find the $d_{i k}$ and $C_{k j}$ of InGaN and then estimate its $e_{i j}$ from Eq. (30) (" $e$-last"). The two routes produce significantly different internal electric fields, as shown in Sec. III B.

\section{RESULTS AND DISCUSSION}

\section{A. Effect of electromechanical coupling}

Table II illustrates the effect of introducing electrome- 


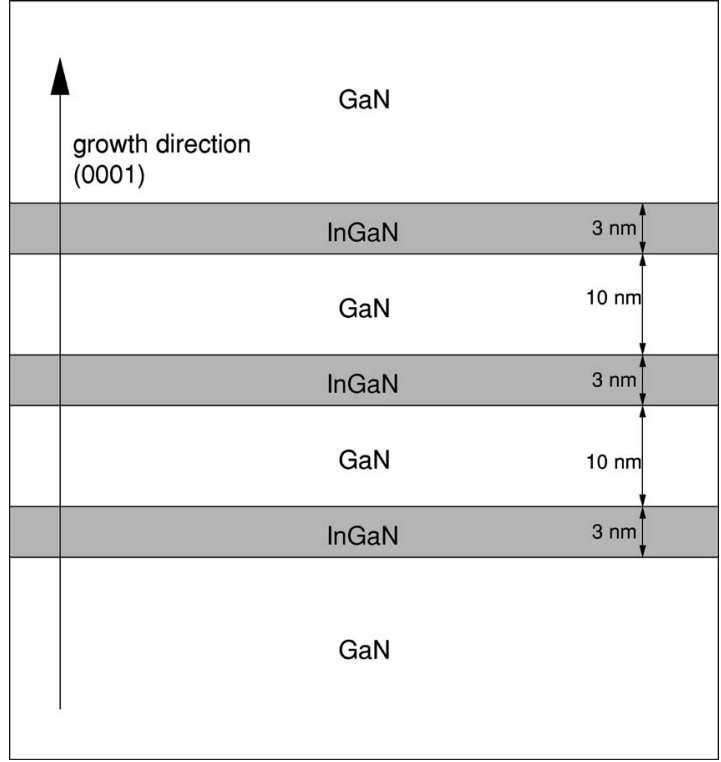

FIG. 1. Schematic of the device used in our examples. The In fraction is 0.2 unless explicitly stated.

chanical coupling using as examples, first, a single $3 \mathrm{~nm}$ QW with the growth direction parallel to the [0001] crystal axis and, second, the MQW stack used by Blume et al. ${ }^{8}$ (illustrated in Fig. 1). In the second example we use Eq. (18) in the uncoupled case and Eq. (24) in the coupled case. In both devices coupling reduces the internal field by $2 \%$ and, as one would expect, it reduces the blueshift (the difference between the ground-state transition energy at zero bias and the maximum ground-state transition energy, when the applied field cancels the internal field) by a comparable amount. $E_{\mathrm{tr}, 0}$, the transition energy at zero bias, increases (by about 1\%) because the internal field is reduced.

The consequence of Eq. (25) is that there is a small positive strain in the growth direction in the barrier, of the order of $2 \times 10^{-4}$, two orders of magnitude smaller than that in the well. Using Eq. (25) instead of Eq. (15) reduces the strain in the growth direction in the well by about $6 \%$. The effect is to increase the ground-state transition energy by $0.2 \%$ and the blueshift by $1 \%$. This is a small secondary effect and we neglect it in all calculations that follow, using instead Eq. (15) and assuming the barrier to be fully relaxed.

\section{B. e-first or e-last?}

The $e$-first and $e$-last routes described in Sec. II D do not produce the same results, as Figs. 2-5 show. Here we have used as an example the device depicted in Fig. 1. For an In fraction of 0.2 , using $e$-last instead of $e$-first increases the internal field by $9 \%$, reduces $E_{\mathrm{tr}, 0}$ by about $2 \%$ and increases the blueshift $\Delta E_{\text {tr }}$ (the difference between the transition energy at zero bias and the maximum transition energy, when the applied field is equal and opposite to the internal field) by $10 \%-15 \%$.

To test the accuracy of the $e$-first and $e$-last routes, we simulated various devices in literature. Table III shows our calculations of $F, E_{\mathrm{tr}, 0}$, and $\Delta E_{\mathrm{tr}}$ using the $e$-last route (in

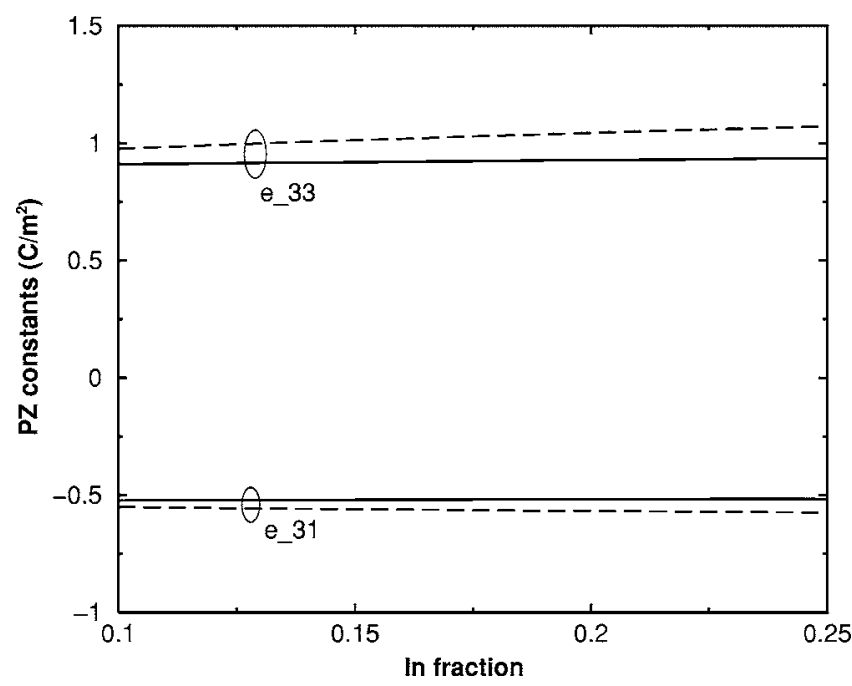

FIG. 2. PZ constants $e_{31}$ and $e_{33}$ calculated by the $e$-first (solid line) and $e$-last (dashed line) routes.

italics) and the $e$-first route (in bold font). Our $e$-first values of $F$ and $\Delta E_{\mathrm{tr}}$ correspond more closely with literature values than do our $e$-last results.

Which route is more logical? $e$-last involves the product of two linearly interpolated quantities $\left(d_{i j}\right.$ and $\left.C_{k l}\right)$ and is therefore quadratic in $x$. This is surprising for what is ostensibly a linear interpolation approach. With $e$-first, $e_{i j}$ is a linear function of $x$, as we would expect. $e$-first gives results which are closer to the experimental results. There is evidence that the In in InGaN tends to form lumps or dots, ${ }^{30}$ which means that it is sensible to calculate the properties of the binaries so far as possible before combining them. This would favor using $e$-first.

Altogether, $e$-first is preferable and we use it henceforth. However, we have not found any discussion of the alternative routes in literature.

\section{Band profile and wave functions}

We now present some sample output from our model, taking as an example the $\mathrm{In}_{0.2} \mathrm{Ga}_{0.8} \mathrm{~N} / \mathrm{GaN}$ device illustrated

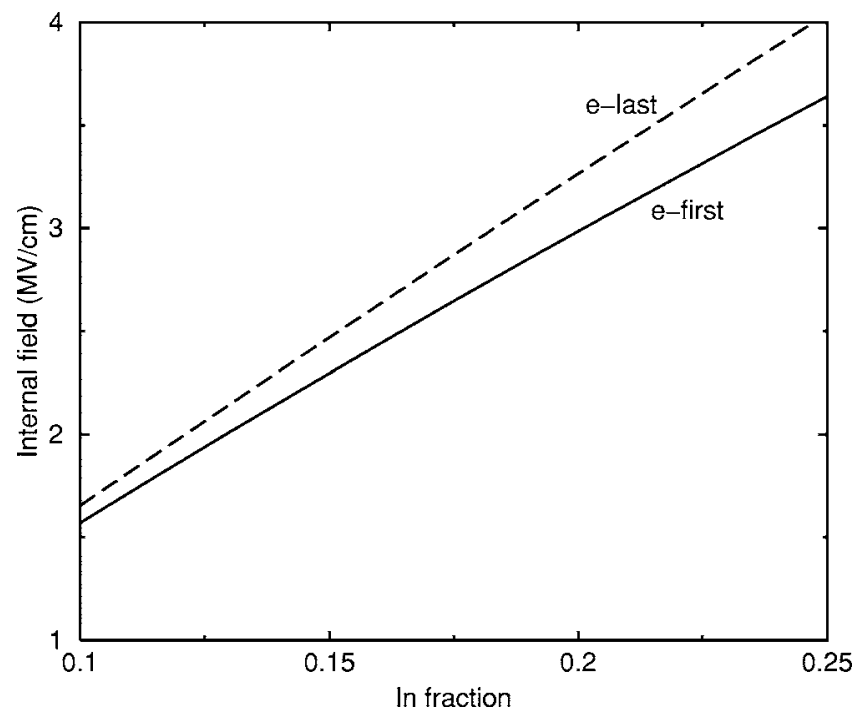

FIG. 3. Internal field as a function of In fraction, using two different methods of calculating the PZ constants $e_{i j}$. 


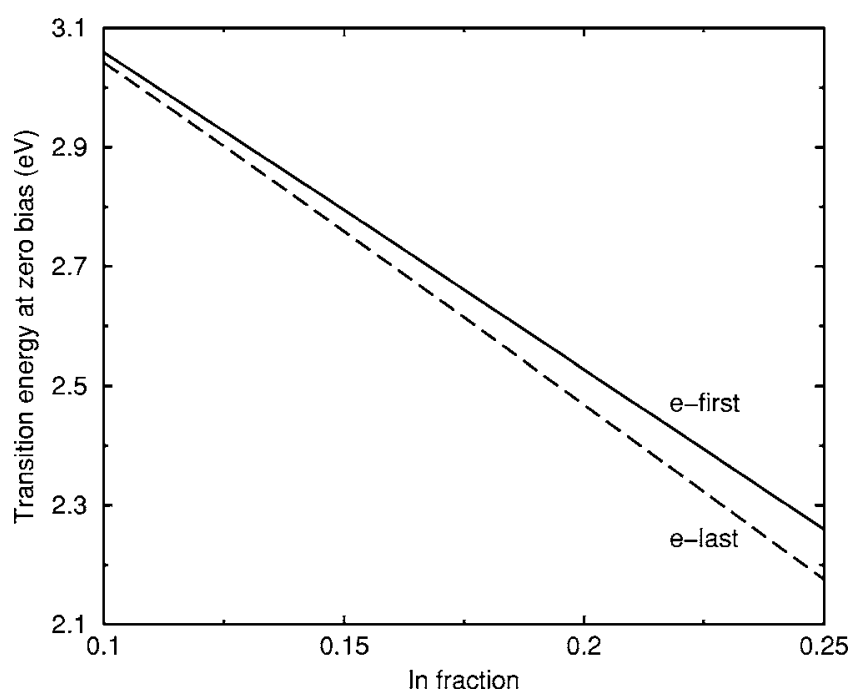

FIG. 4. Transition energy at zero bias $\left(E_{\mathrm{tr}, 0}\right)$, as a function of In fraction, using two different methods of calculating the PZ constants $e_{i j}$.

in Fig. 1. Figure 6(a) shows the band profile with no applied bias, together with the ground-state electron and hole wave functions, illustrating the spatial separation of electrons and holes and the redshift due to the internal field. The slope in the barrier is due to the periodic boundary conditions.

Figure 6(b) shows the near-flatband conditions, when a reverse bias approximately equal to the internal field is applied; the transition energy is blueshifted and the overlap between the electron and hole wave functions (and thus the intensity of the light emitted) is maximized (in this case we have applied additional, effectively infinite potential barriers about $1 \mathrm{~nm}$ outside the well edge, to prevent oscillatory wave functions; this does not affect the transition energy).

Figure 7 shows the ground-state transition energy $E_{\mathrm{tr}}$ as a function of reverse bias. The maximum $E_{\mathrm{tr}}$ is at flatband conditions.

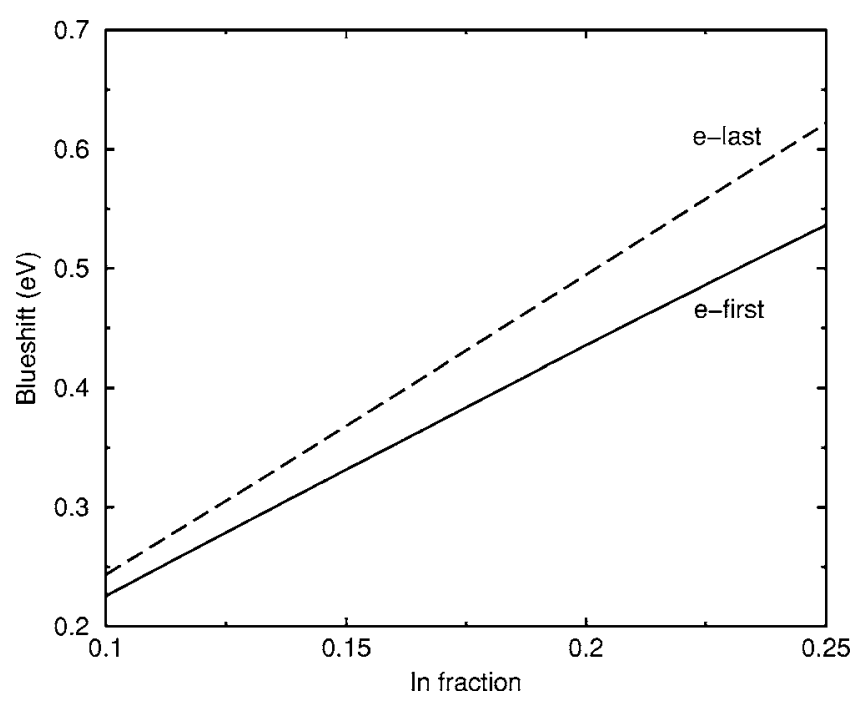

FIG. 5. Blueshift (difference between transition energy at zero bias and at flatband) as a function of In fraction, using two different methods of calculating the PZ constants $e_{i j}$.

\section{Effect of uncertainty in stiffness and PZ constants on internal field and optical properties}

We now consider the most important material parameters that influence the internal field, namely, the elastic stiffness and the PZ constants, and examine their effect on the internal field, and thus on the optical properties, of an $\mathrm{In}_{0.2} \mathrm{Ga}_{0.8} \mathrm{~N} / \mathrm{GaN}$ MQW.

There is a wide range of published values for the elastic stiffness and PZ tensor elements of GaN and InN. Some examples are given in Table IV. The uncertainty surrounding the measurement of the PZ constants is illustrated by the results of Guy et al., ${ }^{31}$ who report two different values of $d_{33}$ - varying by $50 \%$ - from apparently identical samples of InN. A further difficulty is that the so-called PZ constants are actually strain-dependent. ${ }^{32,33}$

Table IV gives (in bold type) the values of $e_{i j}$ calculated from the published values of $d_{i j}$ by using Eq. (30) with Vurgaftman and Meyer $C_{i j}{ }^{11}$ To illustrate the effect on $e_{i j}$ of the uncertainty in $C_{i j}$, Table IV also presents extreme values of $e_{i j}$ calculated from the highest and lowest values of $C_{i j}$ (marked $h$ and $l$ in the table). The error bars on $e_{i j}$ are of the order of $50 \%$ (as much as $75 \%$ in the case of the lower limit of the $e_{33}$ of $\left.\mathrm{GaN}\right)$.

Such uncertainty in the input parameters is bound to give rise to uncertainty in the optical properties. $C_{i j}$ affects the internal field in two separate ways: through the calculation of $e_{i j}$ [Eq. (30)] and through the calculation of strain [Eq. (13)]. We now take some sample high and low values (for GaN) of each parameter in turn (marked $h$ and $l$ in Table IV) and investigate the effect on $F, E_{\mathrm{tr}, 0}$, and $\Delta E_{\mathrm{tr}}$ of perturbing the input value (we do not consider uncertainties in InN parameters, since InN typically only forms $20 \%$ of the alloy, so $\mathrm{GaN}$ properties dominate).

Once again we use as an example the device depicted in Fig. 1, with coupled periodic boundary conditions. As can be seen from Table $\mathrm{V}$, uncertainties in $e_{i j}$ give rise to a deviation of up to $+26 \%$ or $-23 \%$ in the internal field and of as much as $+38 \%$ or $-32 \%$ in blueshift. The direct effect of uncertainty in $C_{i j}$ on the results is smaller, giving rise to an variation of the order of $\pm 10 \%$ or less. The effect of uncertainties in $e_{i j}$ on transition energy is smaller still, producing an uncertainty of no more than about $\pm 5 \%$, while the effect of $C_{i j}$ on electronic transition energy is minimal (less than 1\%).

In summary, uncertainties in the values of $e_{i j}$ (due in part to uncertainties in the values of $C_{i j}$ ) cause significant uncertainties in the optical properties of InGaN/GaN QWs. These are large compared to the differences between the electromechanically coupled and uncoupled models. In the light of this, the use of an electromechanically coupled model may not be warranted for this system.

\section{E. Internal field, $E_{\mathrm{tr}, 0}$, and blueshift}

We used our model to simulate a range of $\mathrm{InGaN} / \mathrm{GaN}$ devices in the literature with various well widths and In fractions and we compared the optical properties we calculated with those reported. Takeuchi et al., ${ }^{4}$ Jho et al., ${ }^{7}$ Waltereit et al., ${ }^{12}$ Feng et al., ${ }^{13}$ and Hangleiter et al. ${ }^{34}$ use photoluminescence to investigate the ground-state optical transition en- 
TABLE III. Comparison between literature values (in approximately chronological order) and present calculations of internal field, transition energy at zero applied field, and blueshift due to applied field. The In fraction $x_{\mathrm{In}}$ and the well width $L_{w}$ are shown, but other details of the structure are not. Italics denote present calculations using the $e$-last route and Vurgaftman and Meyer PZ constants (Ref. 11) (see Table I). Present calculations using the $e$-first route and Vurgaftman and Meyer PZ constants (Ref. 11) are in bold type and present calculations using the $e$-first route and Shimada et al. PZ constants (Ref. 18) in brackets. All literature values are experimental except where indicated.

\begin{tabular}{|c|c|c|c|c|c|c|c|c|c|c|c|c|c|c|}
\hline \multirow{3}{*}{$\frac{\text { Reference }}{4}$} & \multirow{3}{*}{$\frac{x_{\text {In }}}{0.15}$} & \multirow{3}{*}{$\begin{array}{c}\begin{array}{c}L_{w} \\
(\mathrm{~nm})\end{array} \\
3.0\end{array}$} & \multicolumn{4}{|c|}{$\begin{array}{c}\mathrm{F} \\
(\mathrm{MV} / \mathrm{cm})\end{array}$} & \multicolumn{4}{|c|}{$\begin{array}{l}E_{\mathrm{tr}, 0} \\
(\mathrm{eV})\end{array}$} & \multicolumn{4}{|c|}{$\begin{array}{l}\Delta E_{\mathrm{tr}} \\
(\mathrm{eV})\end{array}$} \\
\hline & & & \multirow{2}{*}{$\begin{array}{l}\text { lit } \\
1.2\end{array}$} & \multicolumn{3}{|c|}{ present calc } & \multirow[t]{2}{*}{ lit } & \multicolumn{3}{|c|}{ present calc } & \multirow[t]{2}{*}{ lit } & \multicolumn{3}{|c|}{ present calc } \\
\hline & & & & 2.2 & 2.0 & (1.5) & & & & & & & & \\
\hline & 0.16 & 3.0 & 1.2 & 2.3 & 2.1 & (1.6) & 2.81 & 2.78 & 2.82 & $(2.91)$ & 100 & 315 & 280 & (175) \\
\hline \multirow[t]{2}{*}{6} & 0.23 & 3.0 & 1.7 & 3.7 & 3.4 & (2.6) & 2.76 & 2.29 & 2.37 & $(2.53)$ & 150 & 570 & 495 & (335) \\
\hline & 0.23 & 2.0 & 1.9 & 3.8 & 3.4 & (2.6) & 2.87 & 2.67 & 2.71 & $(2.80)$ & 50 & 300 & 255 & (165) \\
\hline \multirow[t]{2}{*}{7} & 0.15 & 2.2 & 2.1 & 2.6 & 2.4 & (1.8) & 2.72 & 2.93 & 2.96 & $(3.04)$ & 100 & 255 & 230 & (140) \\
\hline & 0.20 & 3.5 & & & & & 2.45 & 2.18 & 2.26 & $(2.48)$ & & & & \\
\hline \multirow[t]{2}{*}{12} & 0.085 & 4.2 & & & & & 2.71 & 3.01 & 3.03 & $(3.12)$ & & & & \\
\hline & 0.119 & 4.2 & & & & & 2.57 & 2.81 & 2.85 & $(2.96)$ & & & & \\
\hline 16 (theory) & 0.15 & 3.0 & & & & & 2.81 & 2.55 & 2.60 & $(2.79)$ & & & & \\
\hline \multirow[t]{3}{*}{13} & 0.11 & 3.0 & & & & & 2.9 & 3.03 & 3.04 & $(3.12)$ & & & & \\
\hline & 0.16 & 3.0 & & & & & 2.75 & 2.76 & 2.79 & $(2.90)$ & & & & \\
\hline & 0.21 & 3.0 & & & & & 2.66 & 2.48 & 2.54 & $(2.67)$ & & & & \\
\hline 14 & 0.16 & 6.0 & & & & & 2.56 & 2.21 & 2.28 & $(2.51)$ & & & & \\
\hline 35 & 0.18 & 3.0 & & & & & 2.9 & 2.65 & 2.69 & $(2.81)$ & & & & \\
\hline \multirow[t]{2}{*}{15} & 0.11 & 2.4 & & & & & 3.05 & 3.10 & 3.12 & (3.18) & & & & \\
\hline & 0.11 & 3.3 & & & & & 2.99 & 2.98 & 3.00 & (3.09) & & & & \\
\hline 17 (theory) & 0.16 & 3.0 & 2.4 & 2.4 & 2.2 & (1.6) & & & & & & & & \\
\hline 8 & 0.20 & 3.0 & 0.6 & 3.3 & 3.0 & $(2.2)$ & 2.77 & 2.47 & 2.53 & (2.68) & 50 & 495 & 435 & (285) \\
\hline
\end{tabular}

ergies and/or internal field. Peng et al. ${ }^{35}$ use selective wavelength excitation with photoluminescence, Wang et al. ${ }^{15}$ photoluminescence under high excitation, Lai et al. ${ }^{6}$ electrotransmission, Dhar et al. ${ }^{14}$ cathodoluminescence, and Blume et al. ${ }^{8}$ electromodulated reflectivity to investigate the optical properties. Oriato and Walker ${ }^{16}$ use a coupled Poisson-Schrödinger approach, and Xiao and $\mathrm{Kim}^{17}$ use the Rashba-Sheka-Pikus Hamiltonian. Table III sets out our calculated internal field, $E_{\mathrm{tr}, 0}$, and blueshift together with the corresponding values in the literature. Present calculations are shown in bold type and literature values in ordinary type. $E_{\mathrm{tr}, 0}$ depends not only on In fraction and well width but also on the barrier width and whether the device is a MQW; hence our simulations of devices which have the same $x_{\text {In }}$ and $L_{w}$ (Refs. 4 and 13) do not necessarily produce the same results.

Where internal field is reported in the experimental work, the field is deduced from $E_{\mathrm{tr}}$ (as a function of applied bias in the case of Takeuchi et al. ${ }^{4}$ Lai et al. ${ }^{6}$ and Jho et al., ${ }^{7}$ and as a function of well width in the case of Hangleiter et $\left.a l .{ }^{34}\right)$. Our internal fields and blueshifts tend to be on the high side (although the match with another theory paper (Xiao and $\mathrm{Kim}^{17}$ ) is reasonably good). This may be partly due to screening, which is not taken into account in our model. Or it may be that the PZ constants we use are too high. We have redone the calculations (in brackets in Table III) using the Shimada et al. (zero-strain) PZ constants, ${ }^{18}$ which were calculated $a b$ initio and which produce a better fit with literature values of internal field and blueshift (it is not possible to use his strain-dependent PZ tensor ${ }^{32}$ directly, as volume is not conserved). The PZ constants of Bykhovski et al. ${ }^{36}$ are even lower than those of Shimada et al. and could potentially produce an even better fit to literature results, but they are estimated from the PZ constants of zincblende GaN and they do not seem reliable so they have not been used here.

The discrepancies between the published values and present calculations of $E_{\mathrm{tr}, 0}$ are of the order of $\pm 0.2 \mathrm{eV}$. While there is significant uncertainty in this area, our values fall somewhere in the middle of the range. Our values based on Shimada et al. ${ }^{18}$ correspond slightly less well with the literature values than do our values based on Vurgaftman and Meyer; ${ }^{11}$ the average differences between the present and literature values are +0.08 and $-0.04 \mathrm{eV}$, respectively. However, taking the table as a whole, the Shimada-based PZ constants give better agreement with the experiment.

Figure 8 shows our values of $E_{\mathrm{tr}, 0}$ as a function of well width for various In fractions, first using the Vurgaftman and Meyer PZ constants ${ }^{11}$ from Table I, then using the Shimada et al. (zero-strain) values. ${ }^{18}$ In each case we have assumed a stack of quantum wells with barriers of width $10 \mathrm{~nm}$.

To illustrate the amount of uncertainty in emission energies from InGaN devices, we show in Fig. 9 a subset of data from Table III: $E_{\mathrm{tr}, 0}$ versus In fraction for cases where the well width is $3 \mathrm{~nm}\left(E_{\mathrm{tr}, 0}\right.$ depends also on barrier width and whether the device is a MQW). The literature values are shown together with our calculations, using first the standard Vurgaftman and Meyer PZ constants ${ }^{11}$ from Table I, and then those of Shimada et al. ${ }^{18}$ The table indicates that the Shimada-based PZ constants tend to give the best agreement with literature values, particularly where $x_{\mathrm{In}}>0.16$. 


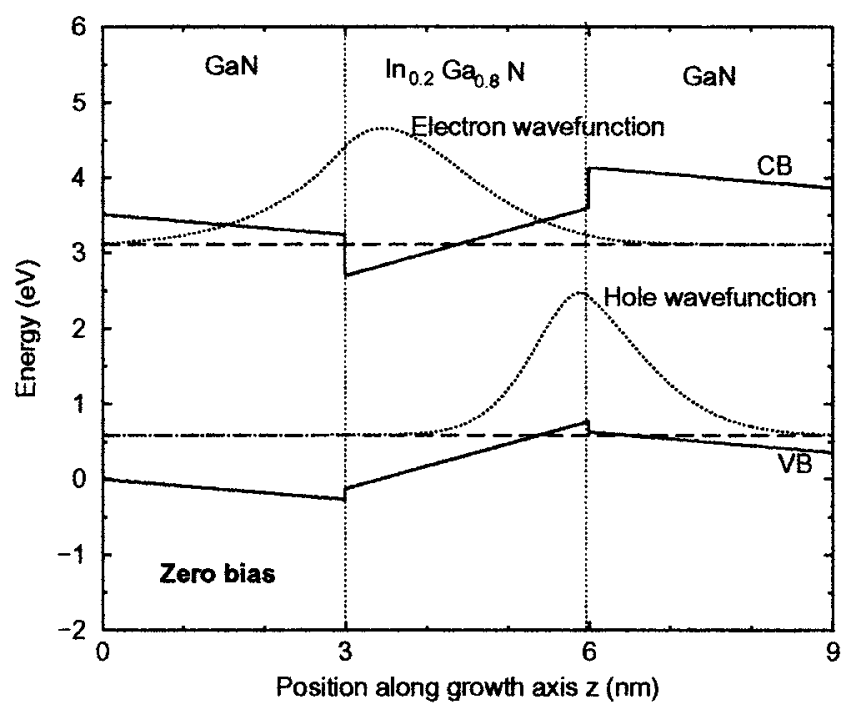

(a)

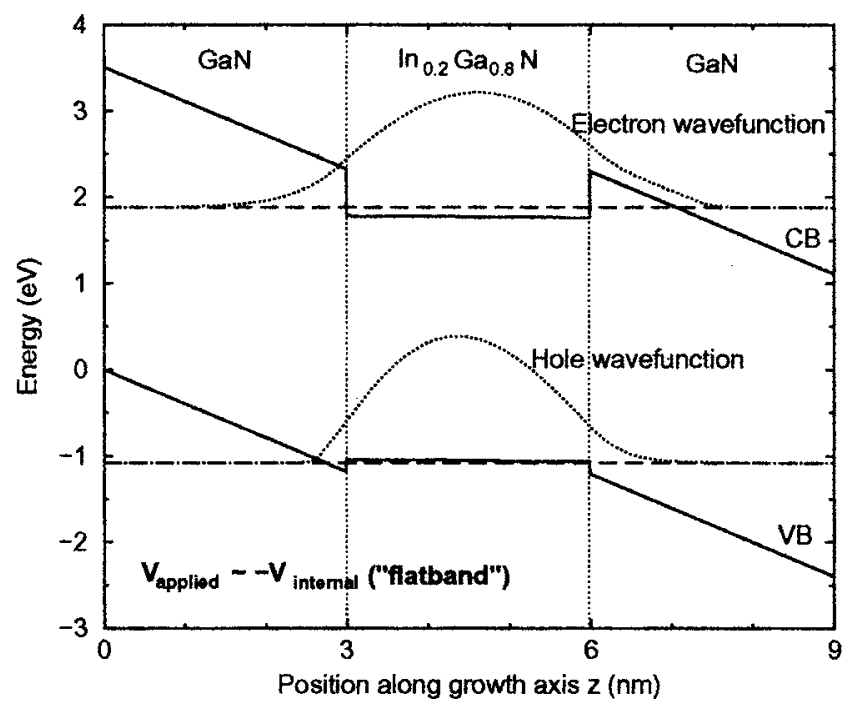

(b)

FIG. 6. Example of band profile (solid lines), electron and hole ground state energies (dashed lines), and electron and hole wave functions (dotted lines) in an $\mathrm{In}_{0.2} \mathrm{Ga}_{0.8} \mathrm{~N} / \mathrm{GaN} \mathrm{MQW}$ with well width of $3 \mathrm{~nm}$ and barrier width of $10 \mathrm{~nm}$, (a) at zero bias and (b) when the applied field is approximately equal to the internal field (flatband conditions).

We end this subsection by reflecting on some of the issues which may give rise to the variation we have seen between the optical properties of outwardly similar InGaN devices, and between theory and experiment. The properties of a device depend greatly on growth conditions, ${ }^{37,38}$ including reactor design, temperature, growth pressure, carrier gas, and flow rates. They depend also on the choice of substrate and on the thickness of the buffer layer. Interface roughness, the density of threading dislocations, ${ }^{39}$ and that of In clusters, ${ }^{30}$ also affect the optical properties. Discrepancies between theory and experiment arise partly from the difficulty of growing samples of reasonable quality and sufficient size for an accurate measurement of the PZ constants, so it may be that $a b$ initio calculations such as Shimada et al. ${ }^{18}$ are currently more reliable ( $a b$ initio methods can accurately reproduce experimental values of PZ constants for materials such as GaAs where the experimental uncertainties are relatively

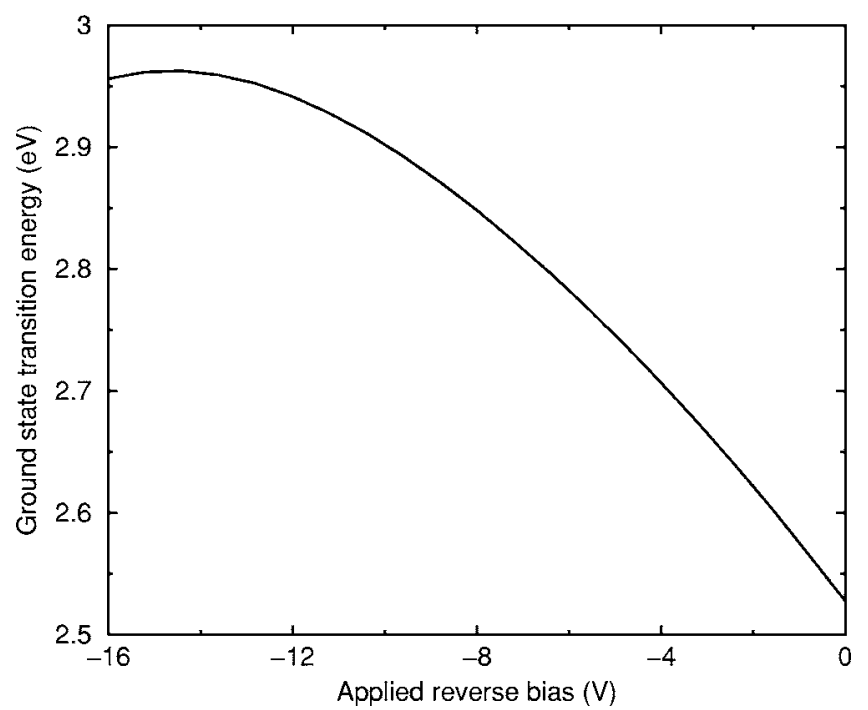

FIG. 7. Typical ground-state transition energy as a function of applied reverse bias.

small $\left.^{40}\right)$. Another difficulty in simulating experimental results is that the dependence of $e_{i j}$ on In fraction is usually assumed to be linear, but there is evidence that it is actually quadratic. $^{22}$ The strain dependence of $e_{i j}$ (Refs. 32 and 33) is an additional complication which is not yet well understood. The field of III nitrides is a young one, where the material properties are not yet fully researched.

\section{F. Effect of In gradient on $E_{\mathrm{tr}, 0}$ and blueshift}

The In content may not be constant across a well, partly because the higher temperature needed to grow the second GaN barrier may cause some of the In to boil off. We now investigate how an In gradient in the growth direction affects the optical properties. We take four arbitrary profiles, constant, linear, quadratic, and quartic, all with the same average In content, and one quadratic profile with a different average In content, as shown in Fig. 10. For ease of computation, we varied the In fraction in 20 discrete steps across the well, rather than in a continuous manner.

Figure 11 shows the band profiles corresponding to the first four In profiles. While the well bottom becomes more and more curved as the degree of the polynomial increases, this is a mere detail compared to the feature which dominates all the scenarios: the slope in the well due to the PZ field. Thus the ground-state electron and hole energies and the transition energies do not differ significantly between different profiles.

Figure 12 shows the ground-state transition energy as a function of applied bias, for each of the In profiles pictured. The result is similar for all the profiles having the same average In content; the only one that is noticeably different is the quadratic profile with the same limits as the linear profile, but a smaller average In content. This shows that, while the total In content is an important factor affecting the optical properties, the manner in which it is distributed along the growth axis is not important if the distribution is smooth (we are not discussing In segregation here; the spontaneous formation of In-rich clusters may affect the optical properties). 
TABLE IV. Some published values of elastic and PZ constants, in approximate chronological order, for GaN and InN. Experimental results are marked (e). High and low values used in Table V are marked (h) and (1). Where values of $d_{i j}$ are published, the corresponding values of $e_{i j}$ are calculated by using Eq. (30). The result depends what values of $C_{i j}$ are used; the one using the Vurgaftman and Meyer recommended values (Ref. 11) are shown in bold type, and the $e_{i j} \mathrm{~s}$ calculated from extreme (h) and (1) values of $C_{i j}$ are shown in ordinary type. (d) denotes the value inferred from $d_{33}=-2 d_{31}(\operatorname{Ref} .43)$.

\begin{tabular}{|c|c|c|c|c|c|c|c|c|c|}
\hline & Reference & $\begin{array}{l}C_{11} \\
(\mathrm{GPa})\end{array}$ & $\begin{array}{l}C_{12} \\
(\mathrm{GPa})\end{array}$ & $\begin{array}{l}C_{13} \\
(\mathrm{GPa})\end{array}$ & $\begin{array}{l}C_{33} \\
(\mathrm{GPa})\end{array}$ & $\begin{array}{l}d_{31} \\
(\mathrm{pm} / \mathrm{V})\end{array}$ & $\begin{array}{l}d_{33} \\
(\mathrm{pm} / \mathrm{V})\end{array}$ & $\begin{array}{r}e_{31} \\
\left(\mathrm{C} \mathrm{m}^{-2}\right)\end{array}$ & 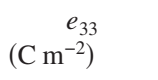 \\
\hline \multirow[t]{31}{*}{$\mathrm{GaN}$} & 36 & & & & & & & -0.22 & 0.43 \\
\hline & 44(e) & $390 \pm 15(\mathrm{~h})$ & $145 \pm 20(\mathrm{~h})$ & $106 \pm 20$ & $398 \pm 20(h)$ & & & & \\
\hline & 45 & 396 & 144 & 100 & 392 & & & & \\
\hline & 46 & 369 & $94.2(1)$ & $66.7(1)$ & 397 & & & & \\
\hline & $47(\mathrm{e})$ & 374 & 106 & 70 & 379 & & & & \\
\hline & 48(e) & 377 & 160 & 114 & 209(1) & & & & \\
\hline & 49(e) & $365 \pm 2$ & $135 \pm 4$ & $114 \pm 16(\mathrm{~h})$ & $381 \pm 1$ & & & & \\
\hline & 50 & 367 & 135 & 103 & 405 & & & & \\
\hline & $51(\mathrm{e})$ & 370 & 145 & 110 & 390 & & & & \\
\hline & 18 & $350(1)$ & 140 & 104 & 376 & & & -0.32 & 0.63 \\
\hline & & & & & & & & $-0.84(\mathrm{~h})$ & 0.28 \\
\hline & 43 & & & & & -1.9 & 3.7 & -0.62 & 1.1 \\
\hline & & & & & & & & -0.36 & $1.3(\mathrm{~h})$ \\
\hline & $52(\mathrm{e})$ & 373 & 141 & 80.4 & 387 & & & & \\
\hline & & & & & & & & -0.68 & 0.24 \\
\hline & 25 and $26(\mathrm{e})$ & & & & & -1.55 & 3.1 & -0.50 & 0.91 \\
\hline & & & & & & & & -0.29 & 1.1 \\
\hline & 22 & & & & & & & -0.42 & 0.57 \\
\hline & 53 & & & 83 & 415 & & & -0.47 & 0.86 \\
\hline & & & & 68 & 354 & & & -0.37 & 0.67 \\
\hline & 27 & & & & & & & -0.37 & 0.67 \\
\hline & & & & & & & & -0.62 & $0.20(1)$ \\
\hline & 23 & & & & & -1.4 & 2.7 & -0.46 & 0.78 \\
\hline & & & & & & & & $-0.27(1)$ & 0.94 \\
\hline & 54 & & & 104 & 414 & & & & \\
\hline & & & & & & & & -0.42 & 0.15 \\
\hline & $34(\mathrm{e})$ & & & & & $-0.96 \pm 0.05$ & $1.92 \pm 0.1(\mathrm{~d})$ & -0.31 & 0.56 \\
\hline & & & & & & & & -0.18 & 0.67 \\
\hline & & & & & & & & -0.71 & 0.23 \\
\hline & 11 & 390 & 145 & 106 & 398 & -1.6 & 3.1 & -0.53 & 0.89 \\
\hline & & & & & & & & -0.31 & 1.1 \\
\hline \multirow[t]{15}{*}{$\mathrm{InN}$} & 45 & 271(h) & 124(h) & 94(h) & $200(1)$ & & & & \\
\hline & 46 & 243 & 71.9(1) & $52.5(1)$ & 263(h) & & & & \\
\hline & 50 & $223(1)$ & 115 & 92 & 224 & & & & \\
\hline & 22 & & & & & & & -0.59 & 0.95 \\
\hline & 53 & & & 88 & 233 & & & -0.56 & 1.09 \\
\hline & & & & 70 & 205 & & & -0.45 & $0.81(1)$ \\
\hline & & & & & & & & $-0.98(\mathrm{~h})$ & 0.86 \\
\hline & 23 & & & & & -3.5 & 7.6 & -0.48 & 1.1 \\
\hline & & & & & & & & $-0.32(1)$ & $1.6(\mathrm{~h})$ \\
\hline & $55(\mathrm{e})$ & & & & & & & -0.90 & 0.66 \\
\hline & (thin film) & & & & & $-3.12 \pm 0.10$ & $6.24(\mathrm{~d})$ & -0.48 & 0.82 \\
\hline & & & & & & & & -0.33 & 1.3 \\
\hline & & & & & & & & -1.0 & 0.76 \\
\hline & $34(\mathrm{e})$ & & & & & $-3.6 \pm 0.5$ & $7.2 \pm 1.0(\mathrm{~d})$ & -0.55 & 0.95 \\
\hline & & & & & & & & -0.38 & 1.5 \\
\hline
\end{tabular}


TABLE IV. (Continued.)

\begin{tabular}{|c|c|c|c|c|c|c|c|c|}
\hline Reference & $\begin{array}{l}C_{11} \\
(\mathrm{GPa})\end{array}$ & $\begin{array}{l}C_{12} \\
(\mathrm{GPa})\end{array}$ & $\begin{array}{l}C_{13} \\
(\mathrm{GPa})\end{array}$ & $\begin{array}{l}C_{33} \\
(\mathrm{GPa})\end{array}$ & $\begin{array}{l}d_{31} \\
(\mathrm{pm} / \mathrm{V})\end{array}$ & $\begin{array}{l}d_{33} \\
(\mathrm{pm} / \mathrm{V})\end{array}$ & $\begin{array}{c}e_{31} \\
\left(\mathrm{C} \mathrm{m}^{-2}\right)\end{array}$ & $\begin{array}{r}e_{33} \\
\left(\mathrm{C} \mathrm{m}^{-2}\right)\end{array}$ \\
\hline \multirow{3}{*}{11} & \multirow{3}{*}{223} & \multirow{3}{*}{115} & \multirow{3}{*}{92} & \multirow{3}{*}{224} & \multirow{3}{*}{-3.5} & \multirow{3}{*}{7.6} & -0.98 & 0.86 \\
\hline & & & & & & & -0.48 & 1.1 \\
\hline & & & & & & & -0.32 & 1.6 \\
\hline
\end{tabular}

\section{CONCLUSIONS}

We have presented electromechanically coupled analytic expressions for the strain and internal field in a quantum well, incorporating spontaneous polarization. We have extended this to incorporate periodic boundary conditions in multiple quantum wells. We find that electromechanical coupling, when used instead of the uncoupled model, reduces the internal field in $\operatorname{In}_{0.2} \mathrm{Ga}_{0.8} \mathrm{~N} / \mathrm{GaN}$ QWs by $2 \%$. This is small compared to the errors due to uncertainties in the values of stiffness and PZ constants. We have pointed out two different interpolation routes for calculating the PZ tensor elements $e_{i j}$ of an alloy from the $d_{i j}$ of the binaries. We favor the "e-first" method, which gives a $10 \%$ lower internal field than " $e$-last." Using an effective-mass model, we have investigated the effect of the uncertainty in the PZ and elastic constants on the internal field and ground-state optical transition in an InGaN/GaN quantum well, and found that the range of published values gives rise to an uncertainty in the internal field of more than $\pm 20 \%$, an uncertainty in blueshift due to applied field of more than $\pm 30 \%$, and in ground-state transition energy of less than $\pm 6 \%$. We find wide variation among values of internal field and optical transition energy

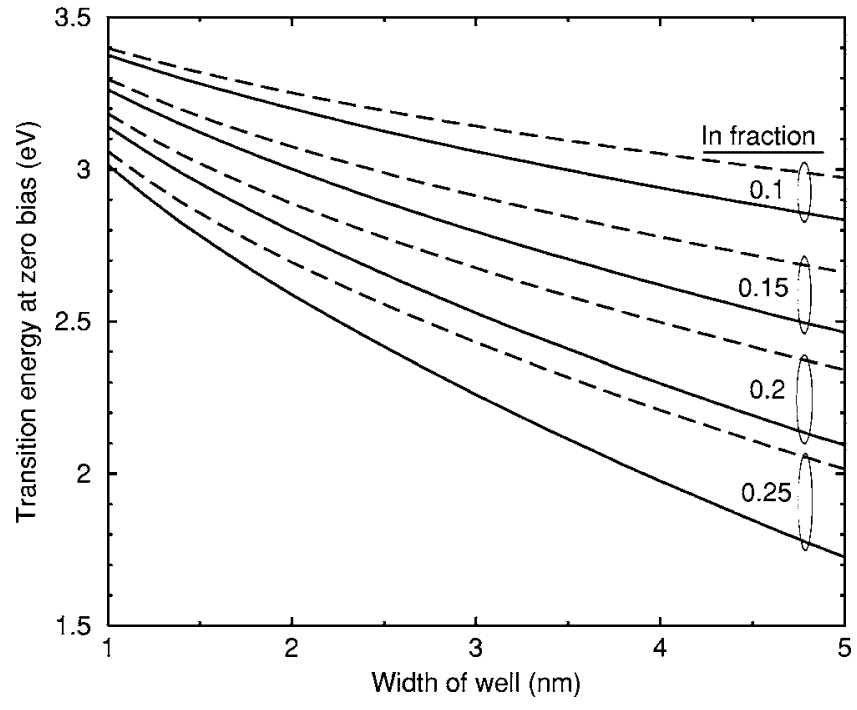

FIG. 8. Ground-state transition energy at zero applied bias $\left(E_{\mathrm{tr}, 0}\right)$, as a function of well width, for various In fractions; using Vurgaftman and Meyer PZ constants (Ref. 11) from Table I (solid lines) and Shimada et al. ${ }^{18}$ (dashed lines).

TABLE V. Effect of uncertainty in stiffness and PZ tensor elements $C_{i j}$ and $e_{i j}$ of GaN on internal field $F$, ground-state optical transition energy at zero applied bias $\left(E_{\mathrm{tr}, 0}\right)$, and blueshift due to applied field $\left(\Delta E_{\mathrm{tr}}\right)$ in an $\mathrm{In}_{0.2} \mathrm{Ga}_{0.8} \mathrm{~N} / \mathrm{GaN}$ MQW with $3 \mathrm{~nm}$ wells and $10 \mathrm{~nm}$ barriers. One parameter at a time is perturbed to its highest or lowest published value and the results are compared with those from Vurgaftman and Meyer recommended parameters (Ref. 11) from Table I; the percentage difference between the recommended and perturbed scenarios is shown.

\begin{tabular}{cccccccrr}
\hline \hline $\begin{array}{c}\text { Parameter } \\
\text { perturbed }\end{array}$ & $\begin{array}{c}\text { Extreme } \\
\text { value }\end{array}$ & $\begin{array}{c}\% \\
\text { change }\end{array}$ & $\begin{array}{c}F \\
\left(\mathrm{MV} \mathrm{cm}^{-1}\right)\end{array}$ & $\begin{array}{c}\% \\
\text { change }\end{array}$ & $\begin{array}{c}E_{\mathrm{tr}, 0} \\
(\mathrm{eV})\end{array}$ & $\begin{array}{c}\% \\
\text { change }\end{array}$ & $\begin{array}{c}\Delta E_{\mathrm{tr}} \\
(\mathrm{meV})\end{array}$ & $\begin{array}{r}\% \\
\text { change }\end{array}$ \\
\hline none & & & 2.98 & 0 & 2.528 & 0 & 435 & 0 \\
$C_{11}(\mathrm{GPa})$ & $350^{\mathrm{a}}$ & -10 & 2.81 & -6 & 2.563 & +1 & 400 & -8 \\
& $405^{\mathrm{b}}$ & +4 & 3.05 & +2 & 2.514 & -0.6 & 450 & +3 \\
$C_{12}(\mathrm{GPa})$ & $94^{\mathrm{c}}$ & -35 & 2.76 & -7 & 2.573 & +2 & 390 & -10 \\
& $165^{\mathrm{b}}$ & +14 & 3.07 & +3 & 2.510 & -0.07 & 455 & +5 \\
$C_{13}(\mathrm{GPa})$ & $66.7^{\mathrm{c}}$ & -37 & 3.09 & +4 & 2.539 & +0.4 & 470 & +8 \\
& $130^{\mathrm{d}}$ & +23 & -2.89 & -3 & 2.526 & -0.1 & 410 & -6 \\
$C_{33}(\mathrm{GPa})$ & $209^{\mathrm{e}}$ & -47 & 2.88 & -3 & 2.463 & -3 & 390 & -10 \\
& $418^{\mathrm{b}}$ & +5 & 2.99 & +0.3 & 2.532 & +0.2 & 440 & +1 \\
$e_{31}\left(\mathrm{C} \mathrm{m}{ }^{-2}\right)$ & $-0.27^{\mathrm{f}}$ & -49 & 2.29 & -23 & 2.666 & +5 & 295 & -32 \\
& $-0.81^{\mathrm{g}}$ & +54 & 3.75 & +26 & 2.365 & -6 & 600 & +38 \\
$e_{33}\left(\mathrm{C} \mathrm{m}^{-2}\right)$ & $0.2^{\mathrm{f}}$ & -78 & 2.50 & -16 & 2.625 & +4 & 335 & -23 \\
& $1.3^{\mathrm{g}}$ & +45 & 3.23 & +8 & 2.476 & -2 & 490 & +13 \\
\hline \hline
\end{tabular}

${ }^{\mathrm{a}}$ Reference 18 .

${ }^{\mathrm{b}}$ Reference 44.

${ }^{\mathrm{c}}$ Reference 46.

${ }^{\mathrm{d}}$ Reference 49.

${ }^{\mathrm{e}}$ Reference 48.

${ }^{\mathrm{f}}$ Reference 23.

${ }^{\mathrm{g}}$ Reference 43. 


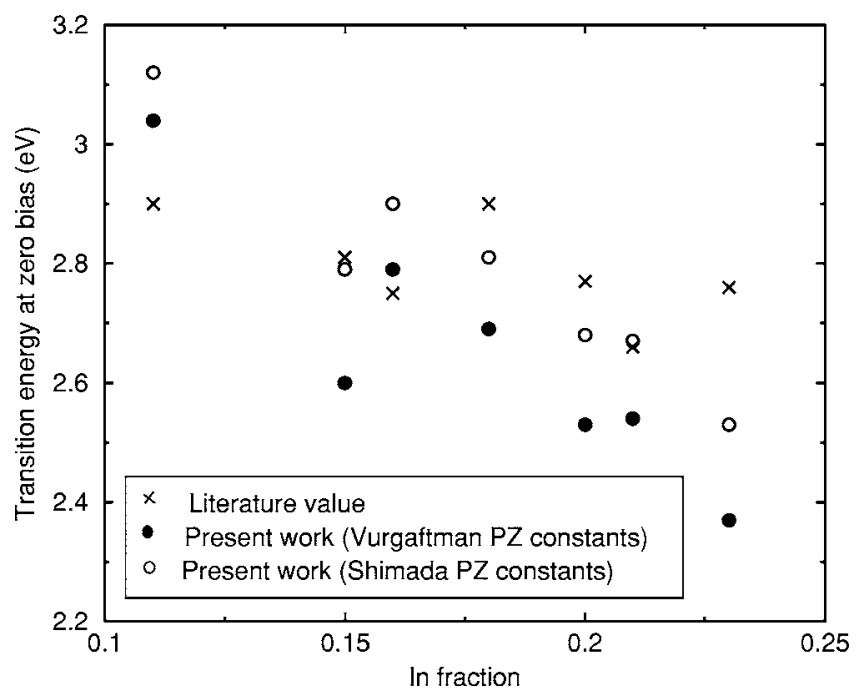

FIG. 9. Comparison of literature values for $E_{\mathrm{tr}, 0}$ (ground-state transition energy at zero applied bias) with those calculated by the present authors for various devices with well width of $3 \mathrm{~nm}$, using standard Vurgaftman and Meyer (Ref. 11) PZ constants (Table I) and Shimada et al. ${ }^{18}$

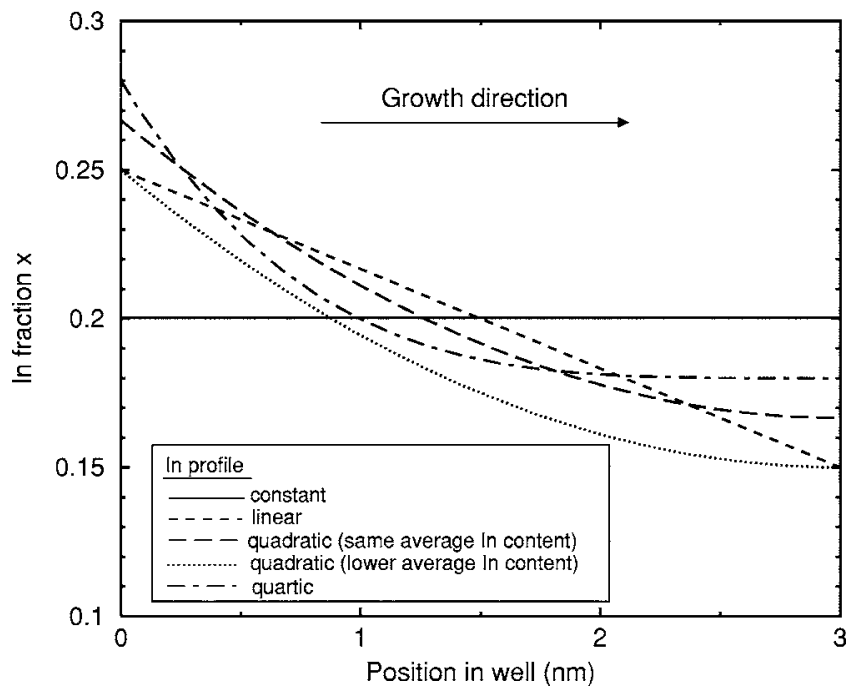

FIG. 10. In profiles used in this investigation. All have the same average In content except the lower of the two quadratic curves, which has the same limits as the linear profile.

of InGaN/GaN devices in the literature, even when the devices are ostensibly similar. This is due to wide variations in growth conditions, defect density, and other factors. Our simulations give values somewhere in the middle of the range. The Shimada et al. ${ }^{18} \mathrm{PZ}$ constants give the best fit to literature values of internal field and blueshift, which suggests that $a b$ initio may be a better means than experiment to find the PZ constants. Finally, we have shown that a QW with a smooth In gradient in the growth direction has similar optical properties to a well with the same average In content distributed evenly along the growth axis.

\section{ACKNOWLEDGMENTS}

We would like to thank Bill Christmas, David Lancefield, and Gunnar Blume for helpful discussions and guidance.

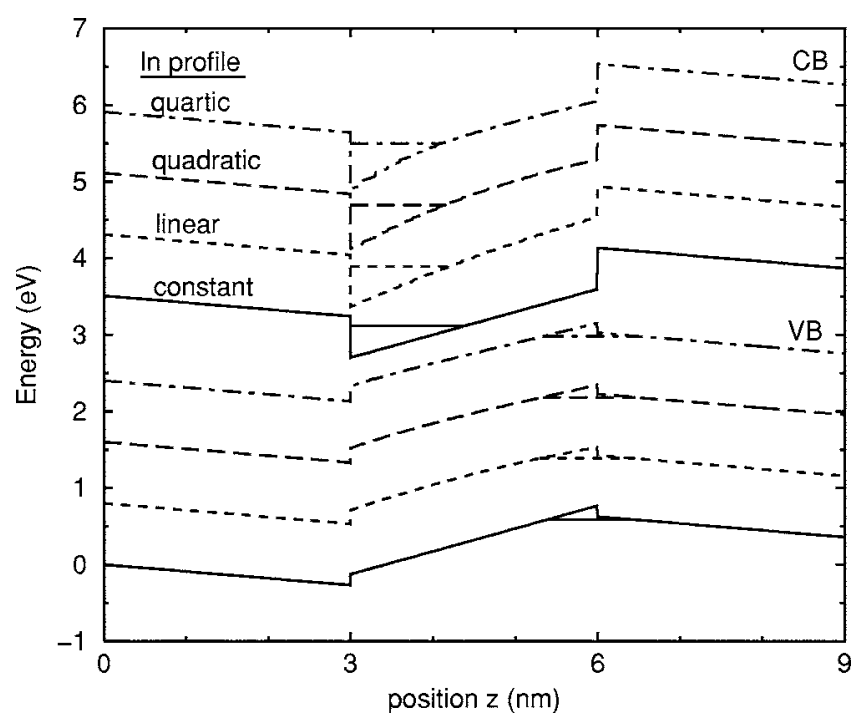

FIG. 11. Band profiles for the various In profiles (excluding the offset quadratic), together with ground-state electron and hole energies.

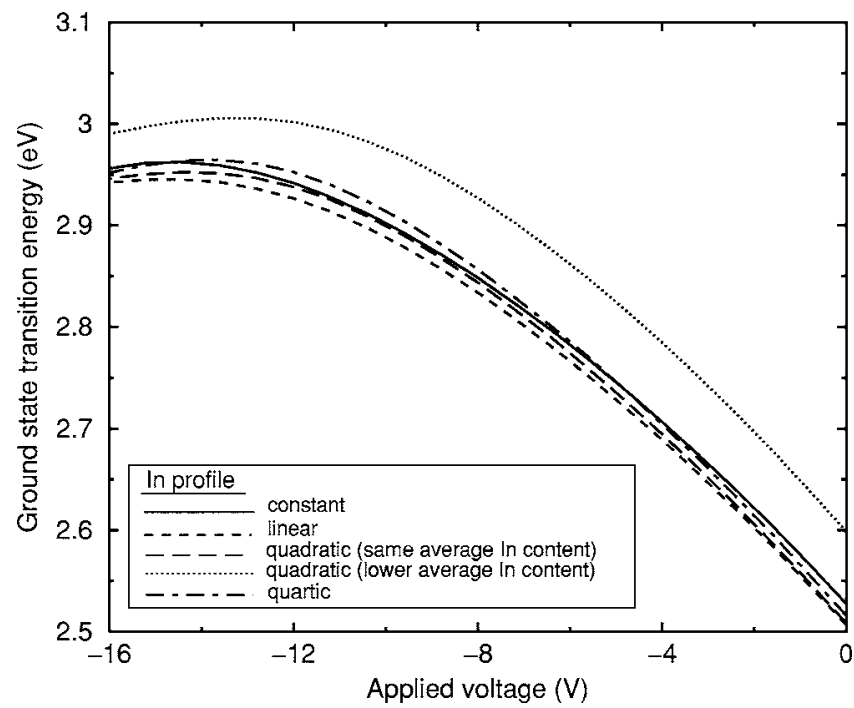

FIG. 12. Ground-state transition energy as a function of applied reverse bias, for various different In profiles along the growth axis: constant, linear, quadratic, and quartic. All have the same average In content except for the quadratic profile producing the upper dotted curve.

${ }^{1}$ Y. Narukawa, I. Niki, K. Izuno, M. Yamada, Y. Murazaki, and T. Mukai, Jpn. J. Appl. Phys., Part 2 41, L371 (2002)

${ }^{2}$ F. Bernardini and V. Fiorentini, Phys. Status Solidi B 216, 391 (1999).

${ }^{3}$ T. Takeuchi, S. Sota, M. Katsuragawa, M. Komori, H. Takeuchi, H. Amano, and I. Akasaki, Jpn. J. Appl. Phys., Part 2 36, L382 (1997).

${ }^{4}$ T. Takeuchi, C. Wetzel, S. Yamaguchi, H. Sakai, H. Amano, and I. Akasaki, Appl. Phys. Lett. 73, 1691 (1998).

${ }^{5}$ P. de Mierry, J. M. Bethoux, H. P. D. Schenk, M. Vaille, E. Feltin, B. Beaumont, M. Leroux, S. Dalmasso, and P. Gibart, Phys. Status Solidi A 192, 335 (2002)

${ }^{6}$ C. Y. Lai, T. M. Hsu, W.-H. Chang, K.-U. Tseng, C.-M. Lee, C.-C. Chuo, and J.-I. Chyi, J. Appl. Phys. 91, 531 (2002).

${ }^{7}$ Y. D. Jho, J. S. Yahng, E. Oh, and D. S. Kim, Phys. Rev. B 66, 035334 (2002).

${ }^{8}$ G. Blume, T. J. C. Hosea, S. J. Sweeney, P. de Mierry, and D. Lancefield, IEE Proc.: Optoelectron. 152, 118 (2005).

${ }^{9}$ E. Pan and F. Tonon, Int. J. Solids Struct. 37, 943 (2000).

${ }^{10}$ E. Pan, J. Appl. Phys. 91, 3785 (2002).

${ }^{11}$ I. Vurgaftman and J. R. Meyer, J. Appl. Phys. 94, 3675 (2003).

${ }^{12}$ P. Waltereit, M. D. Craven, S. P. DenBaars, and J. S. Speck, J. Appl. Phys. 92, 456 (2002).

${ }^{13}$ S.-W. Feng, Y.-C. Cheng, Y.-Y. Chung, and C. C. Chang, J. Appl. Phys. 
92, 4441 (2002)

${ }^{14}$ S. Dahr, U. Jahn, O. Brandt, P. Waltereit, and K. H. Ploog, Appl. Phys. Lett. 81, 673 (2002).

${ }^{15}$ T. Wang, P. J. Parbrook, W. H. Fan, and A. M. Fox, Appl. Phys. Lett. 84, 5159 (2004).

${ }^{16}$ D. Oriato and A. B. Walker, Physica B 314, 59 (2002).

${ }^{17}$ D. Xiao and K. W. Kim, Appl. Phys. Lett. 96, 723 (2004).

${ }^{18}$ K. Shimada, T. Sota, and K. Suzuki, J. Appl. Phys. 84, 4951 (1998).

${ }^{19} \mathrm{P}$. Harrison, Quantum Wells, Wires and Dots: Theoretical and Computational Physics (Wiley, New York, 2000).

${ }^{20}$ P. Ramvall, S. Tanaka, S. Nomura, P. Riblet, and Y. Aoyagi, Appl. Phys. Lett. 73, 1104 (1998).

${ }^{21}$ J. Shi, C. Xia, S. Wei, and Z. Liu, J. Appl. Phys. 97, 083705 (2005).

${ }^{22}$ A. Al-Yacoub and L. Bellaiche, Appl. Phys. Lett. 79, 2166 (2001).

${ }^{23}$ F. Bernardini and V. Fiorentini, Appl. Phys. Lett. 80, 4145 (2002).

${ }^{24}$ S. Muensit and I. L. Guy, Appl. Phys. Lett. 72, 1896 (1998).

${ }^{25}$ C. M. Lueng, H. L. W. Chan, C. Surya, W. K. Fong, C. L. Choy, P. Chow, and M. Rosamond, J. Non-Cryst. Solids 254, 123 (1999).

${ }^{26}$ C. M. Lueng and L. W. Chan, J. Appl. Phys. 88, 5360 (2000).

${ }^{27}$ F. Bernardini, V. Fiorentini, and D. Vanderbilt, Phys. Rev. B 63, 193201 (2001).

${ }^{28}$ K. Osamura, S. Naka, and Y. Murakami, J. Appl. Phys. 46, 4342 (1975).

${ }^{29}$ O. Ambacher et al., J. Phys.: Condens. Matter 14, 3399 (2002).

${ }^{30}$ N. Duxbury, U. Bangert, P. Dawson, E. J. Thrush, W. Van der Stricht, and K. Jacobs, Appl. Phys. Lett. 76, 1600 (2000).

${ }^{31}$ I. L. Guy, Z. Zheng, M. Wintrebert-Fouquet, K. S. A. Butcher, P. Chen, and T. L. Tansley, J. Cryst. Growth 269, 72 (2004).

${ }^{32}$ K. Shimada, T. Sota, K. Suzuki, and H. Okumura, Jpn. J. Appl. Phys., Part 2 37, L1421 (1998).

${ }^{33}$ G. Vaschenko et al., Phys. Status Solidi B 235, 238 (2003).

${ }^{34}$ A. Hangleiter, F. Hitzel, S. Lahmann, and U. Rossow, Appl. Phys. Lett. 83, 1169 (2003).

${ }^{35}$ L.-H. Peng, C.-W. Shih, and C.-M. Lai, Appl. Phys. Lett. 82, 4268 (2003).

${ }^{36}$ A. D. Bykhovski, V. V. Kaminski, M. S. Shur, Q. C. Chen, and M. A.
Khan, Appl. Phys. Lett. 68, 818 (1996).

${ }^{37}$ O. Ambacher, J. Phys. D 31, 2653 (1998).

${ }^{38}$ R. Oliver, Mat. Sci. Technol. 18, 1257 (2002).

${ }^{39}$ H. B. Yu, H. Chen, D. Li, J. Wang, Z. G. Xing, X. H. Zheng, Q. Huang, and J. M. Zhou, J. Cryst. Growth 266, 455 (2004).

${ }^{40}$ J. B. McKitterick, Phys. Rev. B 28, 7384 (1983).

${ }^{41}$ F. Bernardini, V. Fiorentini, and D. Vanderbilt, Phys. Rev. Lett. 79, 3958 (1997).

${ }^{42}$ S. K. Pugh, D. J. Dugdale, S. Brand, and R. A. Abram, Semicond. Sci. Technol. 14, 23 (1999).

${ }^{43}$ I. L. Guy, S. Muensit, and E. M. Goldys, Appl. Phys. Lett. 75, 4133 (1999).

${ }^{44}$ A. Polian, M. Grimsditch, and I. Grzegory, J. Appl. Phys. 79, 3343 (1996).

${ }^{45}$ K. Kim, W. R. L. Lambrecht, and B. Segall, Phys. Rev. B 53, 16310 (1996).

${ }^{46}$ T. Azuhata, T. Sota, and K. Suzuki, J. Phys.: Condens. Matter 8, 3111 (1996).

${ }^{47}$ Y. Takagi, M. Ahart, T. Azuhata, T. Sota, K. Suzuki, and S. Nakamura, Physica B 219, 547 (1996).

${ }^{48}$ R. B. Schwarz, K. Khachaturyan, and E. R. Weber, Appl. Phys. Lett. 70, 1122 (1997).

${ }^{49}$ M. Yamaguchi, T. Tagi, T. Azuhata, T. Sota, K. Suzuki, S. Chichibu, and S. Nakamura, J. Phys.: Condens. Matter 9, 241 (1997).

${ }^{50}$ A. F. Wright, J. Appl. Phys. 82, 2833 (1997).

${ }^{51}$ C. Deger, E. Born, H. Angerer, O. Ambacher, M. Stutzmann, J. Hornsteiner, E. Riha, and G. Fischerauer, Appl. Phys. Lett. 72, 2400 (1998).

${ }^{52}$ T. Deguchi, D. Ichiryu, K. Sekiguchi, and T. Sota, J. Appl. Phys. 86, 1860 (1999).

${ }^{53}$ A. Zoruddu, F. Bernardini, and P. Ruggerone, Phys. Rev. B 64, 045208 (2001).

${ }^{54}$ J.-M. Wagner and F. Bechsteldt, Phys. Rev. B 66, 115202 (2002).

${ }^{55}$ C. B. Cao, H. L. W. Chan, and C. L. Choy, Thin Solid Films 441, 287 (2003). 